\title{
Non-Destructive Impact Method for Quality Assessment of Horticultural Products
}

\author{
J. FELFÖLDI*, I. KERTÉSZ, D. NAGY AND V. ZSOM-MUHA
}

\begin{abstract}
In the case of organic production the quality assessment of the fruits and vegetables is especially important. Monitoring of the maturation and ripening process, early detection of diseases, decision about harvest date and postharvest treatment need reliable, objective and - preferably - non-destructive quality testing methods. Dynamic hardness or stiffness measurement methods (resonance, impact, wave propagation) offer very useful tools in this field, but with strong limitations in applicability area and/or physical interpretation of the measured parameters. Our objective was to develop method and appropriate portable instrumentation to measure surface hardness - as quality measure - with a nondestructive method.

The computer controlled instrument has an electromagnetically excited impactor fitted with a piezoelectric acceleration sensor, a signal conditioner and A/D converter. To ensure the uniform contact behavior (contact area) between the impactor and the tested produce of wide range of shape, spherical head was applied. Conclusively, Hertz contact theory is to be applied for evaluation of the impact signal. Instead of using empirical "hardness index" - as in the case of several existing instruments - our objective was the physical interpretation of the contact phenomena. The measured acceleration signal was mathematically processed to calculate real physical parameters (force, speed, deformation), and to characterize the process similarly to the widely used texture analyzers, penetrometers. A new hardness parameter - "dynamic elastic modulus" - was introduced. According to the methodological investigations, the measurement was found to be perfectly non-destructive for a wide range of products. Conclusively, the developed method offers a useful tool for quality evaluation of organic horticultural products.
\end{abstract}

Keywords: impact test, non-destructive, firmness, fruit, vegetable, physical interpretation

\section{Introduction}

Research works of latest years offer a wide range of novel methods for quality evaluation of products of organic horticulture, for example new, dynamic methods for non-destructive measurement of mechanical properties of fruits and vegetables. They are really useful tools for monitoring of the maturation and ripening process, early detection of diseases, decision about harvest date and postharvest treatment, etc., but

*Corresponding author. E-Mail: Felfoldi.Jozsef@etk.szie.hu

Szent Istvan University, 14-16 Somloi str., Budapest, Hungary

1786-335X@2017 Akadémiai Kiadó, Budapes 
with strong limitations in applicability area and/or physical interpretation of the measured parameters.

The wave propagation methods measure the velocity or the attenuation of the mechanical waves either in through-transmission, or in echo mode, usually in ultrasonic frequency range. The applicability is limited by the high attenuation of most of the horticultural samples. Only certain fruits or vegetables can be tested by the method. Very successful research was published e.g. by Mizrach (2000), applying surface wave measurement on avocado to evaluate the ripeness in laboratory circumstances, however, no portable instrumentation was reported for application in orchard.

The acoustic response method is known as a perfectly non-destructive test for samples of spherical or elongated shape in a relatively wide stiffness range. Due to its extreme reproducibility (Felföldi and Fekete, 2003), the method is especially suitable for detection of small scale changes due to treatments or during maturation. Methods and results were reported about in-vivo applications in orchards (De Belie et al., 2000, Felföldi and Muha, 2007). However, even in this case, there are factors, excluding the application of this resonance based method for given fruits (too soft, pulpy consistency, difficult internal structure, extreme size or shape of seed, etc.). Furthermore, it is necessary to measure other parameters (mass, size) as well for interpretation of the result, resulting in complicated measurement for practical use.

The only dynamic method, with practically no limitations in application field, is the impact method, applying an instrumented impactor to hit the surface of the sample with low kinetic energy and analyzing the contact parameters. The different research groups apply different approaches either in measurement technic or in interpretation of the results.

A number of experimental designs are used for measuring the impact firmness of fruits. One of the applicable experimental designs is the falling impact test (the mechanical properties obtained from dropped fruit impact responses). Based on this type of measurement Cheng-Chang Lien and Ching-Hua Ting (2014) investigated the maturity of the guava. Another possibility is when the impact sensor impacts the fruits to sense of its firmness (García-Ramos et al. (2003)). In case of this technique, impact firmness was used for maturity classification of mango (Padungsak Wanitchang et. al (2011)). Felföldi and Ignát (1999) introduced a dynamic firmness coefficient according to the time to the first peak on the impact curve. For interpretation of the impact signal, a number of parameters were used as well (the maximum of the impact signal, the time to reach the maximum of the signal, slope of the impact curve, area below the curve, 
combination of this parameters, etc.). The common property of these approaches is that the base of the selection of a parameter is the correlation between the given parameter and mechanical characteristics, instead of searching for a physical model behind the impact event.

Our aim was to construct the prototype of a portable impact tester for the organic horticulture, to measure surface hardness - as quality measure with a nondestructive method. Furthermore, our objective was to find the physical interpretation of the impact process, to describe the event with terms of mechanics (force, deformation, speed, energy, etc.). This interpretation is the base of the instrumentation-independent description of the sample properties, and it offers the possibility of comparison of the results of different instruments, different setups too.

\section{Impact Method - Theoretical Considerations}

\subsection{Impactor probe shape}

In the case of a flat impact tip - used in several applications for example in our previous researches (Muha and Felföldi, 2005), the contact area is practically constant during the impact penetration (Fig 1a). Conclusively, the measured force (or acceleration) is proportional to the stress of the tested material; a simple approach can be applied for interpretation of the measured signal. Unfortunately, the flat tip ends with a relatively sharp edge, so in case of a mechanically sensitive tested material, the virtually nondestructive test locally can cause a stress, beyond the bioyield limit of the sample, turning the test to be quasy-nondestructive, as it is illustrated in Fig $1 b$ (one week after the repeated tests of a pear sample).

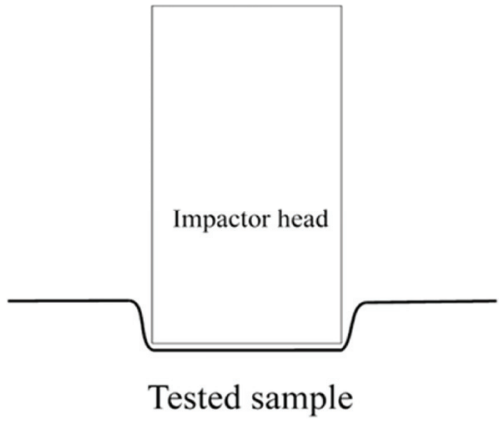

(a)

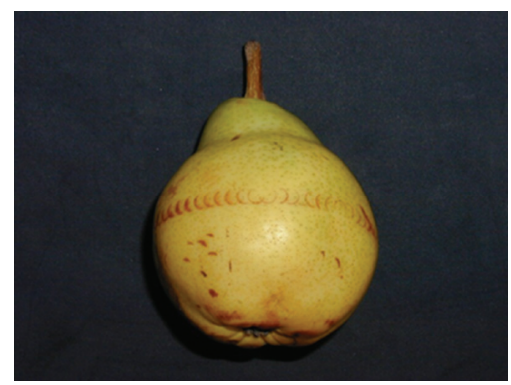

(b)

Figure 1. Deformation of the sample during the test with a flat impactor head (a) and the visible effect of the repeated tests on a pear sample (one week after the tests) 
If the really nondestructive nature of the measurement is a crucial point (invivo tests, application on a sorting line, etc.), a spherical impactor head is more advantageous. There are no sharp edges; the contact is uniform in any relative position of the impactor and the sample, etc. However, in this case, the measured signal is not proportional to the characteristic physical property of the sample (stress), because the contact surface is increasing with the penetration depth (Fig 2). A more sophisticated interpretation of the measured signal is needed, using the Hertz contact theory to describe the penetration process.

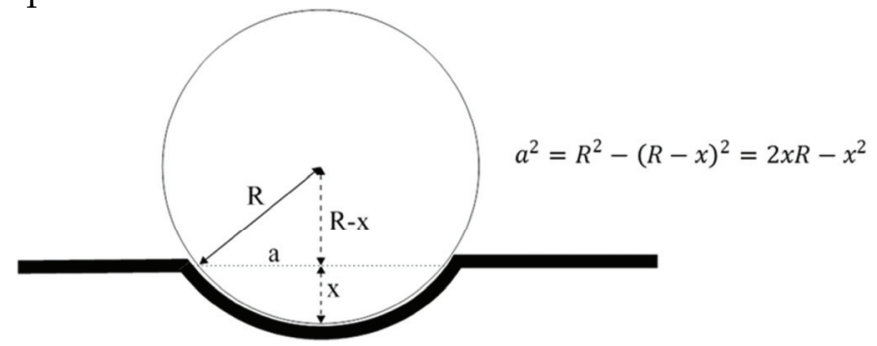

Figure 2. Deformation of the sample during the test with a spherical impactor head and the parameters, used in the assessment

If the impactor head is much smaller, than the sample, the process can be described as a contact between a sphere and a half-space. In this case, according to Mohsenin (1986):

$$
F(x)=\frac{4}{3} E^{*} \sqrt{R} x^{\frac{3}{2}}
$$

where

$$
\begin{aligned}
& -F(x)=: \text { measured force } \\
& -\frac{1}{E^{*}}=\frac{1-\mu_{1}^{2}}{E_{1}}+\frac{1-\mu_{2}^{2}}{E_{2}}
\end{aligned}
$$

and $E_{1}, E_{2}$ are the elastic moduli and $\mu_{1}, \mu_{2}$ the Poisson's ratios associated with the impactor head and the tested sample, respectively

- R: radius of the impactor head

- $x$ : penetration depth

If the impactor tip is rigid $\left(E_{1}>E_{2}\right)$, than

$$
\text { - } \quad E^{*}=\frac{E}{1-\mu^{2}}
$$

and $\mathrm{E}$ is the elastic modulus and $\mu$ is the Poisson's ratio of the sample 


\subsection{Contact force vs. penetration time analysis}

The time-dependency of the contact force during the penetration of the impactor head can be described by differential equations (based on Newton's law of inertia). These equations can be used to evaluate the effect of the different parameters of the measurement on the measured signal, to build a model for interpretation of the measured parameters and connect them to the physical characteristic.

\subsubsection{Elastic materials}

In case of an ideally elastic sample material (the strains are small and within the elastic limit), the contact force is determined only by the mechanical properties of the tested material, the size of the impactor tip and the penetration depth.

$$
F(t)=m \cdot a(t)=m \cdot \frac{d^{2} x(t)}{d t^{2}}
$$

where

- $F(t)$ : force, affecting the impactor (in time)

- $m$ : mass of the impactor

- $a(t)$ : acceleration (practically deceleration) of the impactor due to the resistance of the sample

$-x(t)$ : impactor displacement in time

Combining Eq. (1) and Eq. (2) (taking into account the opposite direction of the displacement and the force):

$$
m \cdot \frac{d^{2} x(t)}{d t^{2}}=-\frac{4}{3} E^{*} \sqrt{R} x(t)^{\frac{3}{2}}
$$

To describe real materials with viscoelastic properties, we have to add a speed-dependent member to Eq. (3). Supposing, that the viscoelastic property can be approximated by an additive stress, proportional to the impactor speed and its result in an additive force, proportional to this stress and the contact surface of the impactor head and the sample:

where

$$
F_{v i s c}(t)=A(t) \cdot \sigma_{v i s c}(t)=A(t) \cdot c \cdot v(t)
$$


- $F_{\text {visc }}(t)$ : speed-dependent force component, affecting the impactor

- $A(t)$ : time- (and displacement-) depending contact area

- $\sigma_{v i s c}(t)$ : speed-dependent stress

- $c$ : material constant of the sample (not equal to, but correlating with the viscosity coefficient, dimension: $\mathrm{Pa} \cdot \mathrm{s} / \mathrm{m}$ )

- $v(t)$ : speed of the impactor

According to Fig. 2, the contact area is:

$$
A(t)=\pi \cdot a^{2}=\pi \cdot\left(2 R x(t)-x(t)^{2}\right)
$$

So the final form of the differential equation is:

$$
m \cdot \frac{d^{2} x(t)}{d t^{2}}=-\frac{4}{3} E^{*} \sqrt{R} x(t)^{\frac{3}{2}}-\pi \cdot\left(2 R x(t)-x(t)^{2}\right) \cdot c \cdot \frac{d x(t)}{d t}
$$

The elastic case can be get back at $c=0$ substitution. This equation can be solved at appropriate boundary conditions $(x(0), \mathrm{v}(0))$ and parameter settings to get the description of the impactor. Numerical solutions were calculated in Mathcad (version 14.0.0.163, 2007 Parametric Technology Corporation).

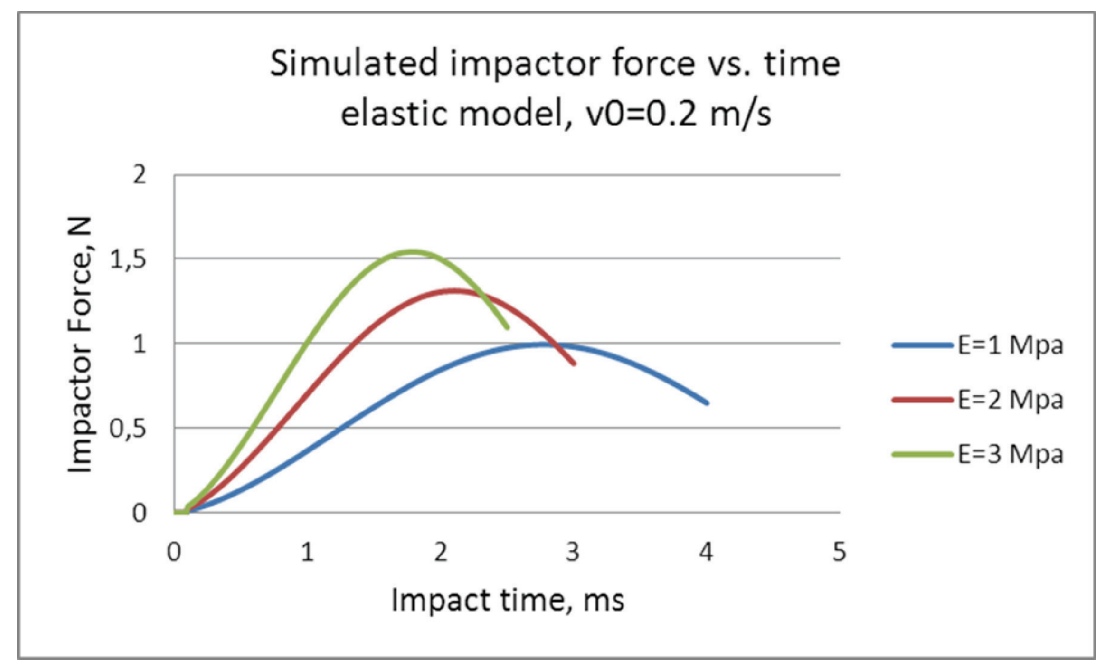

Figure 3. Simulated impactor force vs. time for elastic model (constant impactor speed and different hardness values) 
For the elastic case $(c=0)$, the solutions are illustrated in Figs 3 and 4 , for constant initial impactor speed $(v(0)=0.2 \mathrm{~m} / \mathrm{s})$, constant Poisson's ratio ( $\mu=0.35$, a typical value for horticultural materials, used in all models) and variable elastic coefficient $(E=1,2$ or $3 \mathrm{MPa})$.

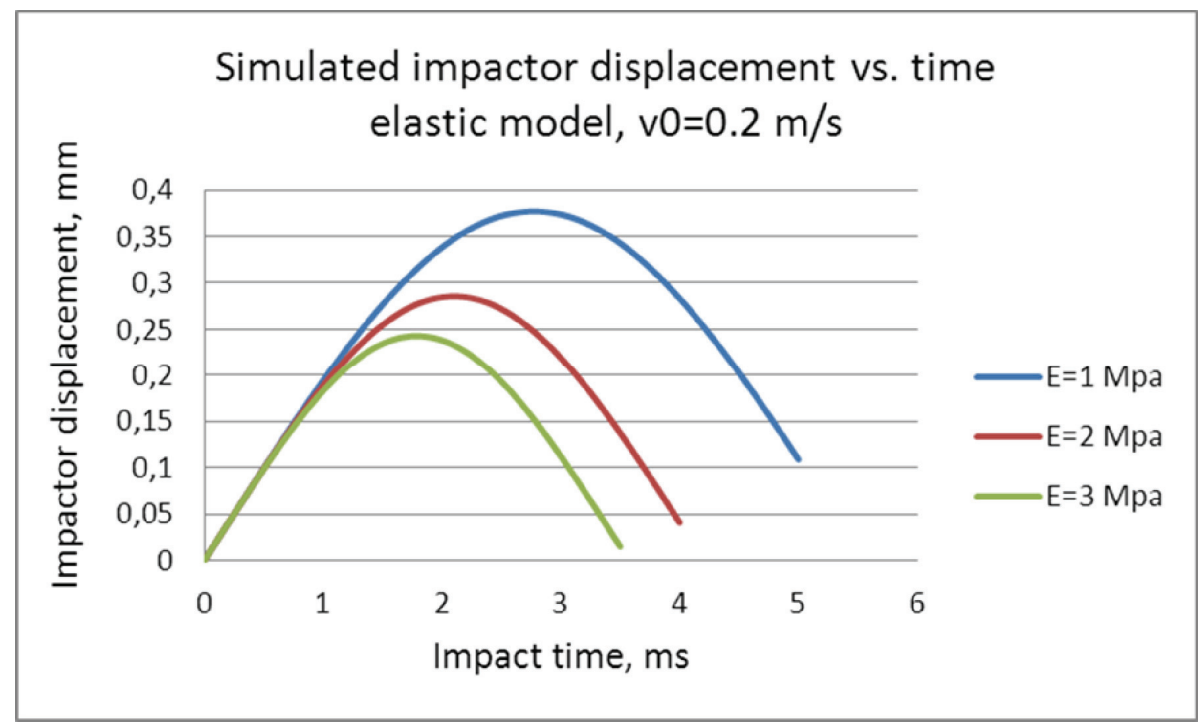

Figure 4. Simulated impactor displacement vs. time for elastic model (constant impactor speed and different hardness values)

The non-linear curvature of the force vs. time is due to the non-linearity in the Hertz-behavior of the spherical impact head. Figures 3 and 4 clearly show that the time, $(d T)$, needed to reach the first peak in displacement or impact force, is decreasing monotonically with the hardness of the sample (characterized by the Young's modulus in the simulation). The simulated behavior validates our former approach, when the sample hardness was estimated based on $d T$-measurement, introducing a "dynamic stiffness coefficient": $d=1 / d T^{2}$ (Felföldi and Ignát, 1999). Furthermore, the simulation results confirmed that for elastic materials the displacement peak time is perfectly coinciding with the force peak time (the maximum force resulted in the maximum penetration) for any speed and hardness levels (illustrated in Fig. 5). 


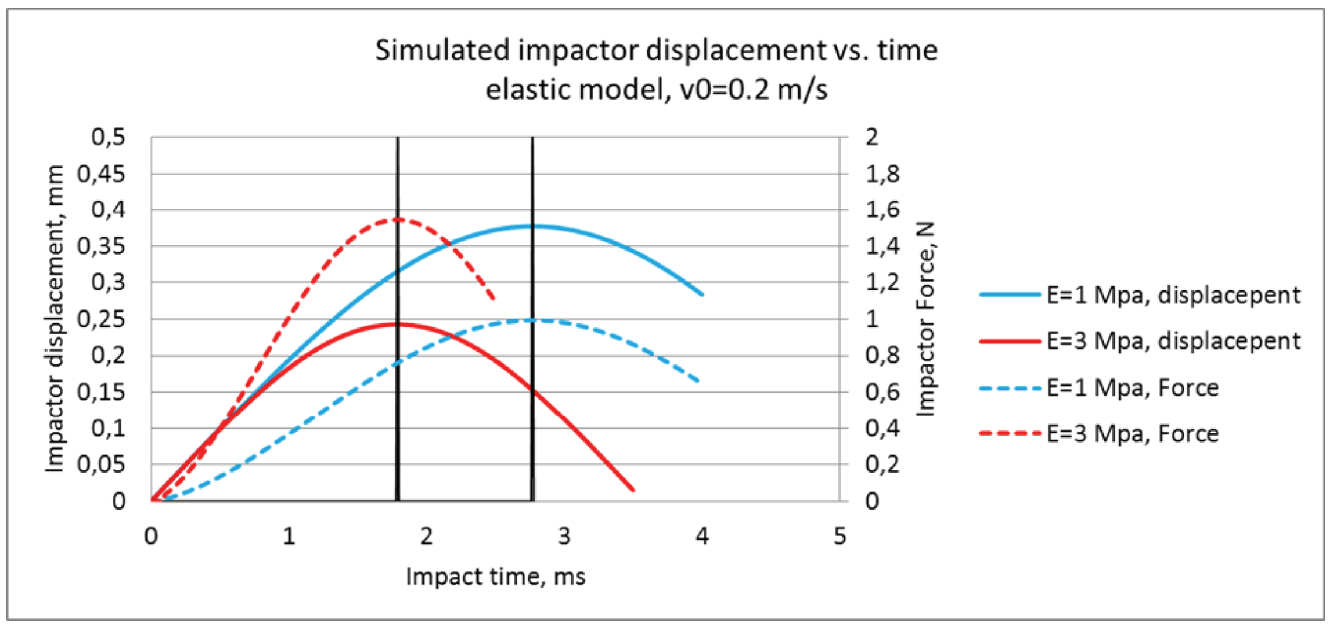

Figure 5. Simulated impactor displacement and force vs. time; highlighted coinciding maxima

However, changing the impactor head from flat to spherical shape (in order of the nondestructive test) resulted in a further consequence: the $d T$ "time to the first peak" became velocity-dependent, as it was shown by the simulation, calculated at different impactor speed levels (Fig. 6). It is caused by the non-linear behavior of the contact force, and needs special attention at the assessment of the measured data later.

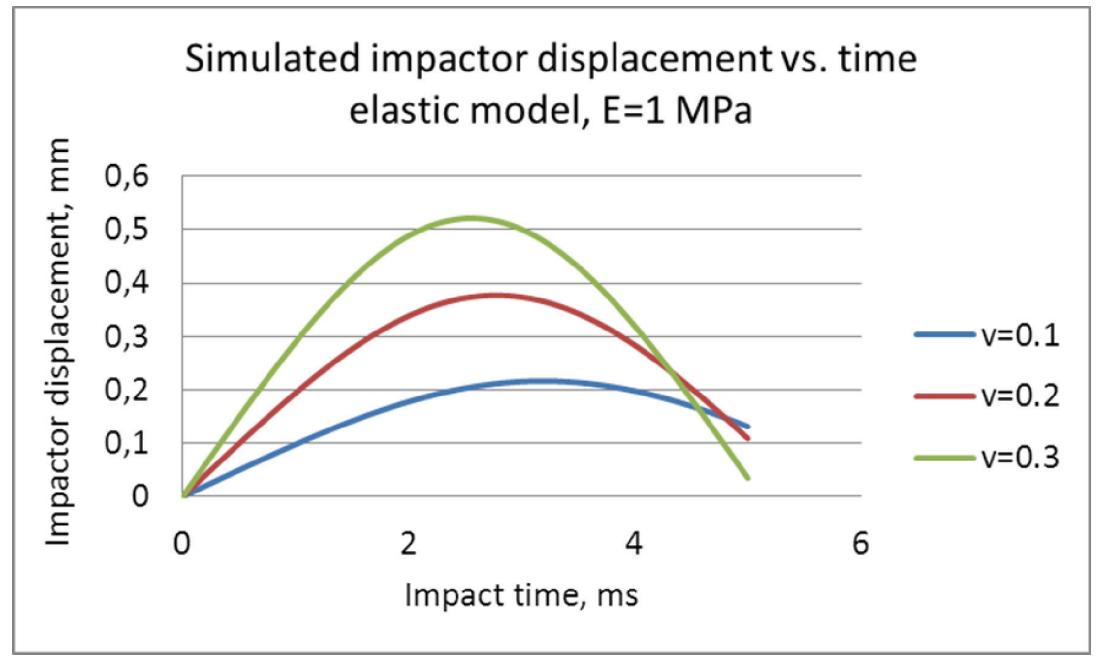

Figure 6. Simulated impactor displacement vs. time for elastic model (constant hardness and different impactor speed values) 


\subsubsection{Viscoelastic materials}

The simulation results described even more difficult behavior of the impactor in case of the viscoelastic nature of the tested materials (taken into account with $c \neq 0$ viscosity constant in Eq. 4). Taking into account the combination of the elastic force increasing with the penetration and the viscous component, affected by the decreasing velocity but increasing contact surface, it is obvious that the behavior can be described only by differential equation and the movement of the impactor is depending on all material and impact parameters. Running the simulation with different boundary conditions $(v(0))$ and material properties $(E, c)$, these dependencies can be investigated and modeled. As an example, Fig. 7 illustrates the impact force (as the measurable parameter) vs. time during the test at $v(0)=0.2 \mathrm{~m} / \mathrm{s}, E=2 \mathrm{MPa}, c=0,10^{6} \mathrm{Pas} / \mathrm{m}$.

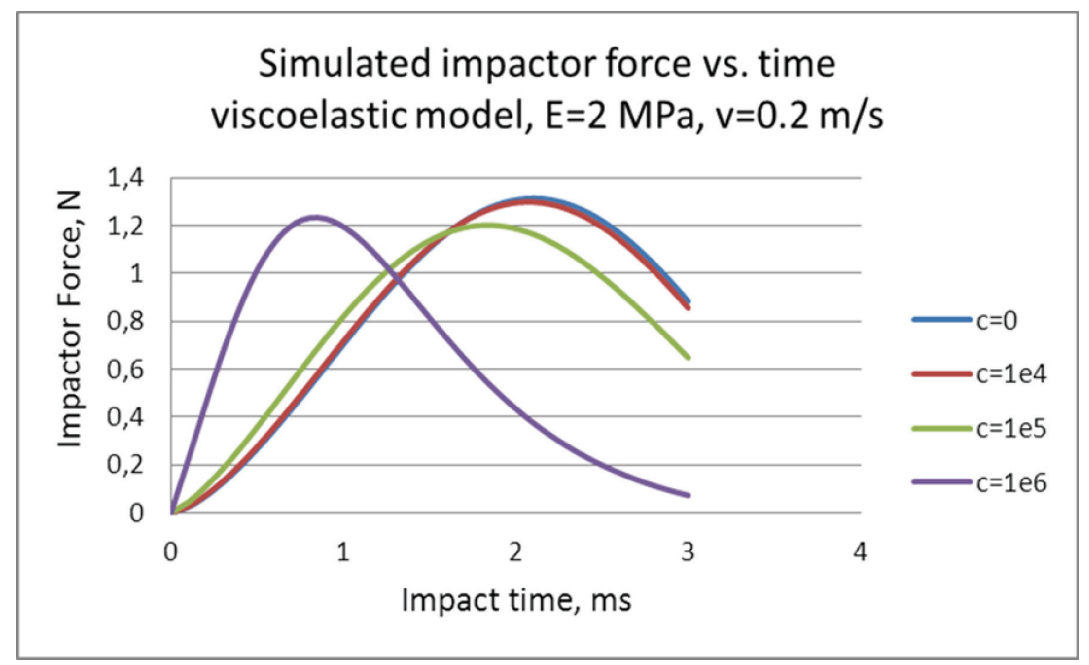

Figure 7. Simulated impactor force vs. time for viscoelastic model (constant impactor speed and hardness values, different viscosity constants)

As Fig. 7 suggests, with these conditions the viscosity has no significant effect (in engineering sense) on the impactor movement below $c=10^{4}$ Pas $/ \mathrm{m}$. However, a viscosity above this level results in a remarkable distortion of the curves either in time, or in amplitude. This conclusion is emphasized in Fig. 8 (same simulation conditions), demonstrating the Force(deformation) function, characterizing the sample mechanical properties in the traditional approach. 


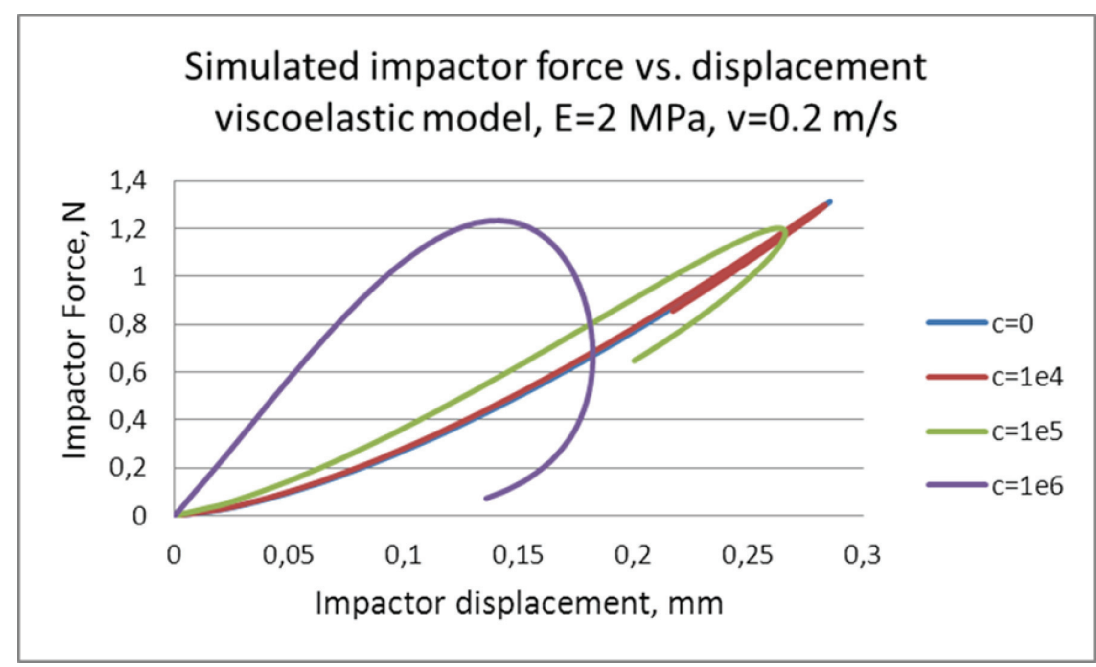

Figure 8. Simulated impactor force vs. deformation for viscoelastic model (constant impactor speed and hardness values, different viscosity constants)

According to the numerical solutions of the differential equation (4), the force vs. displacement curves are overlapping each other below $c=10^{4}$ $\mathrm{Pas} / \mathrm{m}$ viscosity level, and even till $10^{5} \mathrm{Pas} / \mathrm{m}$ level the evaluation seems to be possible with engineering accuracy. It is very encouraging from the point of view of designing a measurement system and developing evaluation methods. However, we have to recognize that the viscosity of the tested material resulted in separating of the "time to the first peak" values on the force and on the displacement curves. It is especially obvious in Fig. 8, $c=10^{6}$ curve: the time of the maximum force and the time of the maximum penetration (zero speed) are different. We have to measure and use different $\mathrm{dT}_{\text {maxForce }}$ and $\mathrm{dT}_{\mathrm{v}=0}$ parameters. This emphasizes the importance of the knowledge of the initial speed of the impactor $(\mathrm{v}(0))$. However, this difficulty is at the same time the key to uncover the type of an unknown sample. Equal $\mathrm{dT}_{\operatorname{maxF}}$ orce and $\mathrm{dT}_{\mathrm{v}=0}$ values mean elastic behavior, while the difference between them shows the level of the viscosity.

Running numerical simulations in a wider range of the parameters $\left(E=2^{*} 10^{5} . .5^{*} 10^{6} \mathrm{MPa}, c=0\right.$.. $\left.2^{*}-10^{5} \mathrm{Pas} / \mathrm{m}\right)$, models can be developed for the estimation of the mechanical characteristics of a sample according to the measured parameters. $\mathrm{D} 1=1 /\left(\mathrm{dT}_{\mathrm{v}=0}\right)^{2}, 1 / \mathrm{ms}^{2}$ and $\mathrm{D} 2=1 /\left(\mathrm{dT}_{\mathrm{Fmax}}\right)^{2}, 1 / \mathrm{ms}^{2}$ parameters were introduced for model building.

For $v(0)=0.2 \mathrm{~m} / \mathrm{s}$ initial impactor speed, a strong correlation was found between the Young's modulus (E) and D1 (Fig. 9) and an acceptable model 
was found to estimate the viscosity constant (c) based on D1-D2 (Fig. 10). These models can be the base of the further investigations and the evaluation of the experimental data.

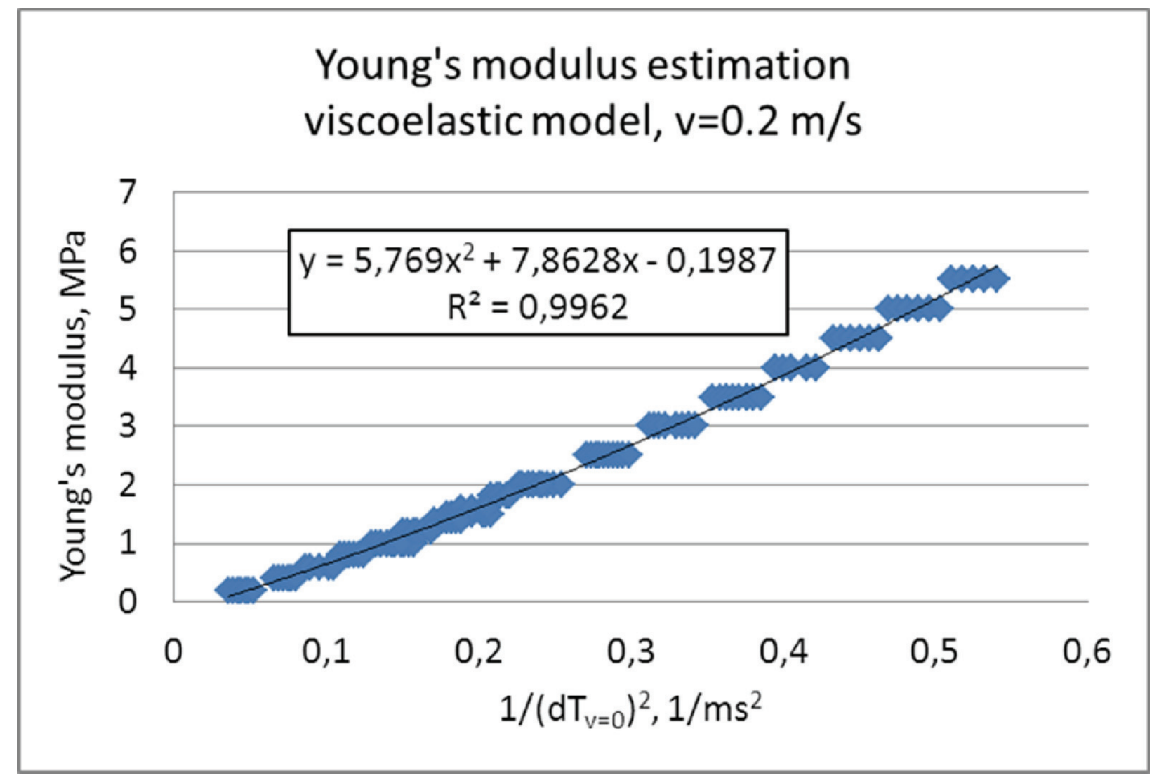

Figure 9. Polynomial model for Young's modulus estimation

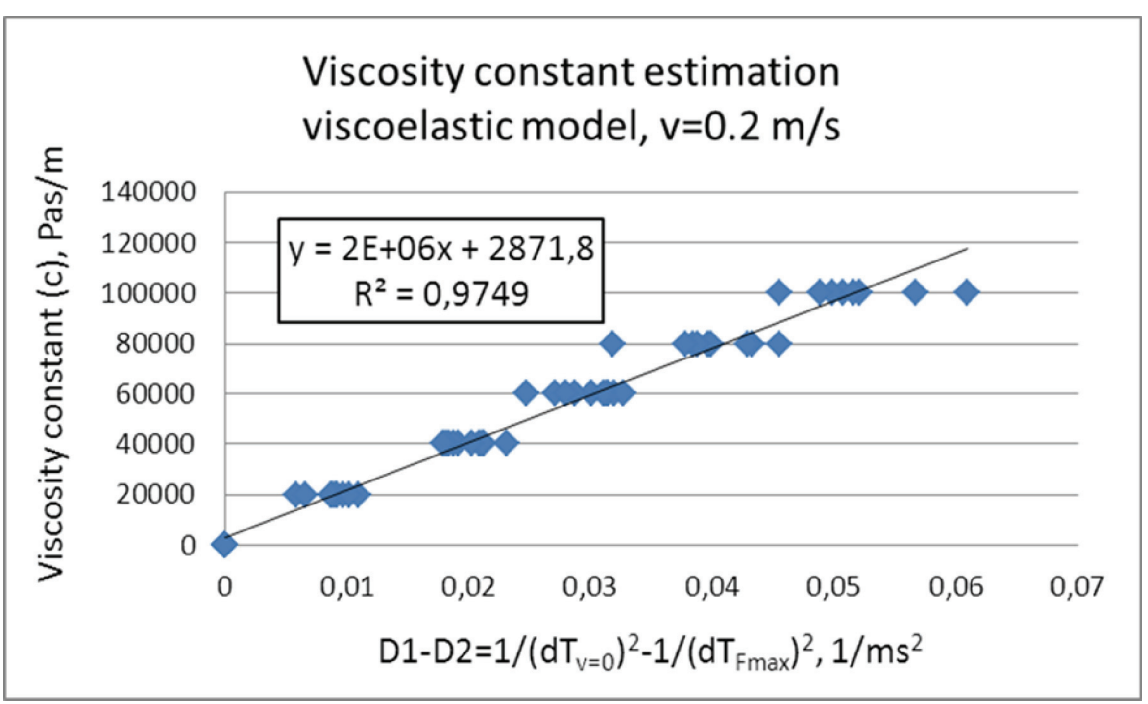

Figure 10. Model for viscosity estimation 


\section{Experimental - Materials and Methods}

\subsection{Impact measurement system}

The base of the measurement system was an electromechanically excited impactor, fitted with a miniature, lightweight $(0.7 \mathrm{~g})$, ceramic shear ICP ${ }^{\circledR}$ accelerometer (PCB Piezotronics, Model 352B10) with sensor characteristics as follows:

- Sensitivity $( \pm 10 \%)$ :

$$
\begin{array}{ll}
\text { - Sensitivity }( \pm 10 \%): & 1.02 \mathrm{mV} /\left(\mathrm{m} / \mathrm{s}^{2}\right) \\
\text { - Measurement Range: } & \pm 4,905 \mathrm{~m} / \mathrm{s}^{2} \mathrm{pk} \\
\text { - Frequency Range }( \pm 5 \%): & 2 \text { to } 10,000 \mathrm{~Hz} \\
\text { - Resonant Frequency } & \geq 65 \mathrm{kHz} \\
\text { - Non-Linearity: } & \leq 1 \%
\end{array}
$$$$
\text { - Resonant Frequency } \quad \geq 65 \mathrm{kHz}
$$

- Non-Linearity:

An ICP signal conditioner was applied to connect the sensor to the A/D converter. In order of the maximum flexibility, a computer-controlled digital storage oscilloscope (Velleman, PCSGU250) was applied to measure the acceleration signal in laboratory circumstances (during the methodological investigations). The measurement range was set from $+/-80 \mathrm{~m} / \mathrm{s}^{2}$ (resolution: $0.3 \mathrm{~m} / \mathrm{s}^{2}$ ) to $+/-2400 \mathrm{~m} / \mathrm{s}^{2}$ (resolution: $10 \mathrm{~m} / \mathrm{s}^{2}$ ), depending on the tested material. The sampling rate was usually $125 \mathrm{kSa} / \mathrm{s}$.

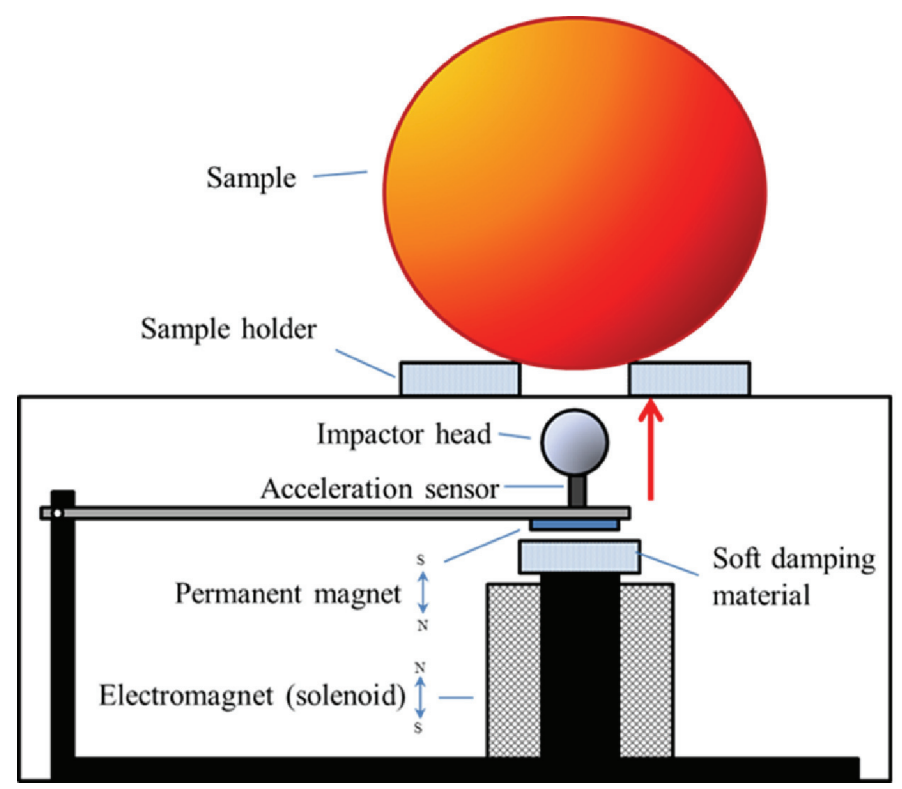

Figure 11. Prototype of the impact measurement instrument 
The sensor was fixed at the end of an impactor arm. The impact movement of the arm - and so the movement of the impact head with the acceleration sensor - was initiated by an electromagnetic solenoid. The energy of the impactor - conclusively the impact speed as well - can be controlled by the excitation voltage applied at the solenoid (Fig. 11).

Spherical impact tip (of $16 \mathrm{~mm}$ diameter) was applied to avoid the uncertain contact surface area and sharp edge effects between the sample and the impactor. It provides with uniform contact behavior (contact area, depending on the displacement of the impact) between the impactor and the tested produce of wide range of shape.

\subsection{Evaluation methods}

Applying a calibrated acceleration sensor, kinetic approach can be applied in physical interpretation of the contact process. The speed of the sensor $(v(t))$ can be calculated integrating the measured acceleration values $(a(t))$ in time, and similarly, the displacement of the impactor $(s(t))$ can be determined, as the integrated value of the speed:

$$
\begin{aligned}
& v(t)=\int_{0}^{t} a(t) d t+v(0) \\
& s(t)=\int_{0}^{t} v(t) d t+s(0)
\end{aligned}
$$

Our final aim was to develop a microcomputer-controlled portable instrument for the measurement, thus different numeric integration methods were compared as well to achieve the reasonable calculation demand with acceptable error. The boundary conditions are crucial questions, as it is typical in differential equation based description of movements. In this case $s(0)=0$, but the initial value of the impactor speed at the contact moment, $v(0)$ is to be determined.

In our simplified approach - resulting in simplified hardware and software needs in instrumentation - we supposed that the time of the maximum deceleration (minimum acceleration), of maximum penetration and of zero speed belong to the very same time $\left(t_{v=0}\right)$.

In this case, eq. (5) can be evaluated in multiple steps: 


$$
v^{\prime}(0)=0 \rightarrow v^{\prime}(t)=\int_{0}^{t} a(t) d t \rightarrow v_{0}=-v^{\prime}\left(t_{v=0}\right) \rightarrow v(t)=v^{\prime}(t)+v_{0}
$$

As it was shown, in case of elastic materials this approach is satisfactory. However, in the case of viscoelastic materials, the maximum penetration and zero speed of the impactor happen really at the same time, but the maximum value of the deceleration of the impactor can be reached earlier. Conclusively, there is no way to describe the impact event exclusively according to the data measured during the impact process; further information about $v_{0}$ is needed. Either preliminary calibration of the hardware or measurement and evaluation of the acceleration phase of the impactor are necessary. However, this second approach needs more sophisticated system (the signal level and duration are very different in these two phases of the movement).

The force $(F)$, decelerating the impactor, characterizing the surface firmness, is proportional to the measured acceleration signal. The coefficient, determined by the inertial behavior of the impactor arm+sensor+impactor tip system, can be substituted by a constant "effective mass" $\left(m^{*}\right)$ value.

$$
F(t)=m^{*} \cdot a(t)
$$

This way, we have the possibility to characterize the mechanical properties of the sample surface similarly to the precision penetrometers: with the Force vs. Deformation relationship. It ensures the possibility of comparison with the traditional, compression based methods and to characterize the samples with parameters of physical dimensions (N, N/mm, Ps, etc.).

The advantage of applying spherical impactor is a uniform contact surface for - practically - any shape and in any position of the sample. However, the disadvantage is that this contact surface is not constant during the impact, it is depending on the momentary penetration depth. Conclusively, the Hertz theory of the normal contact of elastic bodies is to be applied for evaluation of the Force vs Displacement relationship. Applying Eq. (1) for the rigid impactor case:

$$
F(x)=\frac{4}{3} \frac{E}{1-\mu^{2}} \sqrt{R} x^{\frac{3}{2}}
$$

where 
- F: measured force

- E: elastic modulus of the sample

- $\mu$ : Poisson's ratio of the sample

- $R$ : radius of the impactor tip (in our case, it is $8 \mathrm{~mm}$ )

$-x$ : penetration depth

In this approach, the impactor tip is supposed to be rigid and much smaller than the sample. If the impactor radius and sample radius are comparable ( $R_{1}$ and $R_{2}$, respectively),

is to be used in (9).

$$
\frac{1}{R}=\frac{1}{R_{1}}+\frac{1}{R_{2}}
$$

This way, a Dynamic Coefficient of Elasticity ("DCE") parameter can be introduced, including the Poisson number (which is generally not known exactly, but supposed to be $0.35-0.45$ for fruits and vegetables), and connected strictly to the Young's modulus $(E)$ :

$$
D C E=\frac{E}{1-\mu^{2}}=\frac{3}{4 \sqrt{R}} \frac{F(x)}{x^{\frac{3}{2}}}
$$

The dimension of $D C E$ is $\mathrm{MPa}$, if the Force is given in $\mathrm{N}$, and the penetration depth and impactor radius are given in $\mathrm{mm}$.

\subsection{Tested materials}

For methodological investigations of the system, model materials (rubber, elastic foam, etc.) were used to avoid the variability of the material properties. Applicability of the method and instrument for horticultural products (non-destructive nature, repeatability, etc.) was tested on different fruit and vegetable samples in wide size, shape, hardness and structure ranges (apple, nectarine, tomato, bell pepper, etc.). The samples were collected from a local market.

The degree of bruise of the fruit and vegetable samples was evaluated by cutting through the sample at the impacted spot and analyzing the microscopic image of the supposed bruised zone as it was proposed by Rodriguez-Sinovas et al. (1991). 


\subsection{Methodological investigations}

The repeatability of the system was characterized by repeating tests on the same sample, same location, under the same conditions (excitation voltage). At the same time, if the tested sample is a fruit or vegetable, the bruise, caused by the test, can be evaluated, simply with occurrence of a monotonous change in the result during the short-time repetitions.

To characterize the reproducibility, the repeated impact tests were performed on different locations of the tested horticultural sample. In this case, of course, the variability is depending rather on the biological variability of the sample, than on the repeatability of the instrument.

For the data assessment, signal processing, the Mathcad program (version 14.0.0.163, 2007 Parametric Technology Corporation), for statistical calculations Excel (Microsoft Excel, 2010) and SPSS (IBM SPSS Statistics 16) were used.

\section{Results and Discussion}

\subsection{The impactor}

A typical acceleration signal (a) and the impactor speed and displacement values (b), calculated according to the Eq. (7) are shown in Figure 12 (sample: nectarine). Different numeric integration methods were compared as well. Due to the high sample rate and the relative low noise of the system, the trapezoid integration and the Simpson-method gave the same result (so in the following evaluations the simpler trapezoid method was applied).

The usual impact parameters, used by different research groups (time to the first peak, amplitude, area below the curve, etc.) can be read from Fig. 12a, however, the applied approach of physical interpretation provides more information about the process and the given sample:

$-v_{0}=0.236 \mathrm{~m} / \mathrm{s}$

- max. penetration depth $=0.28 \mathrm{~mm}$

- penetration work $=0.56 \mathrm{~mJ}$ 


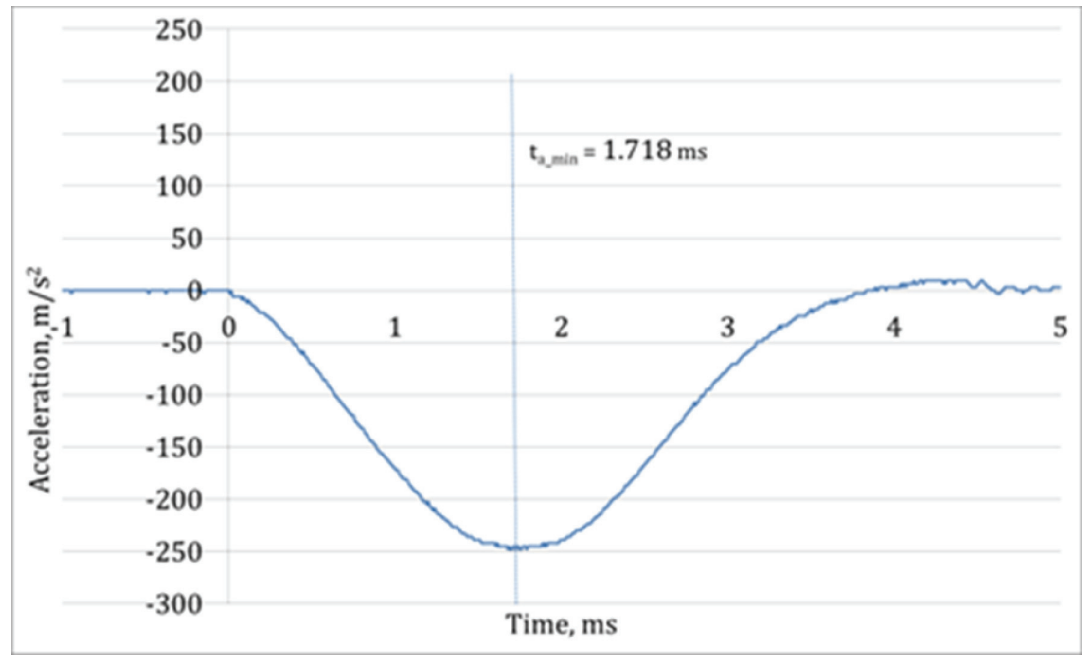

(a)

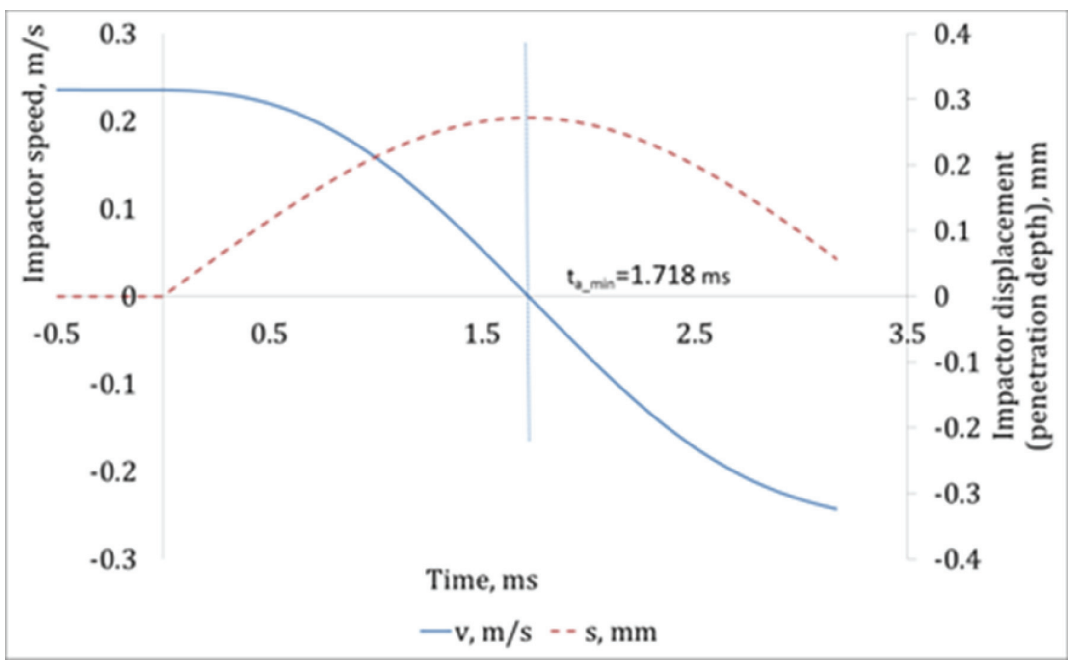

(b)

Figure 12. Typical acceleration signal (a) and the impactor speed and displacement values (b), calculated according to the Eq. (5), (6) and (7) (sample: nectarine)

Furthermore, the force, decelerating the impactor can be calculated according to Eq. (8). With the preliminary calibration, $m^{*}=0.0075 \mathrm{~kg}$ effective mass was determined for the given system. The Force vs Deformation curve of the shown nectarine sample is presented in Figure 13 (a). The DCE parameter can be calculated according to the slope of the (b) curve. 


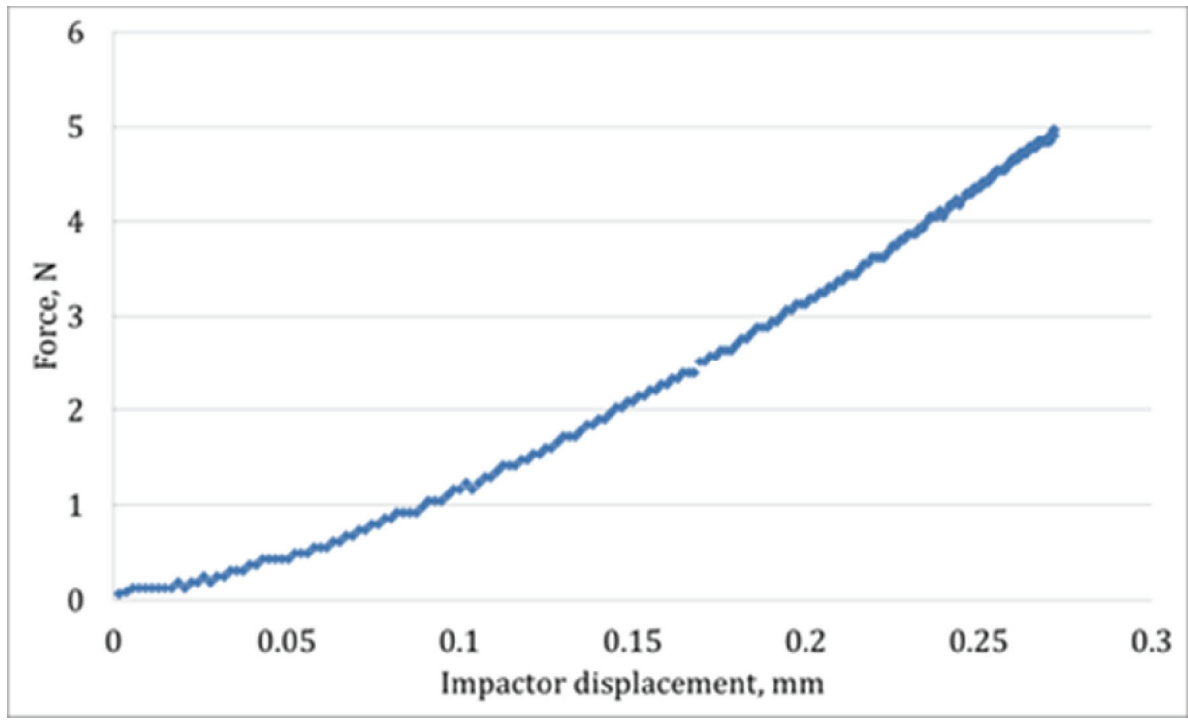

(a)

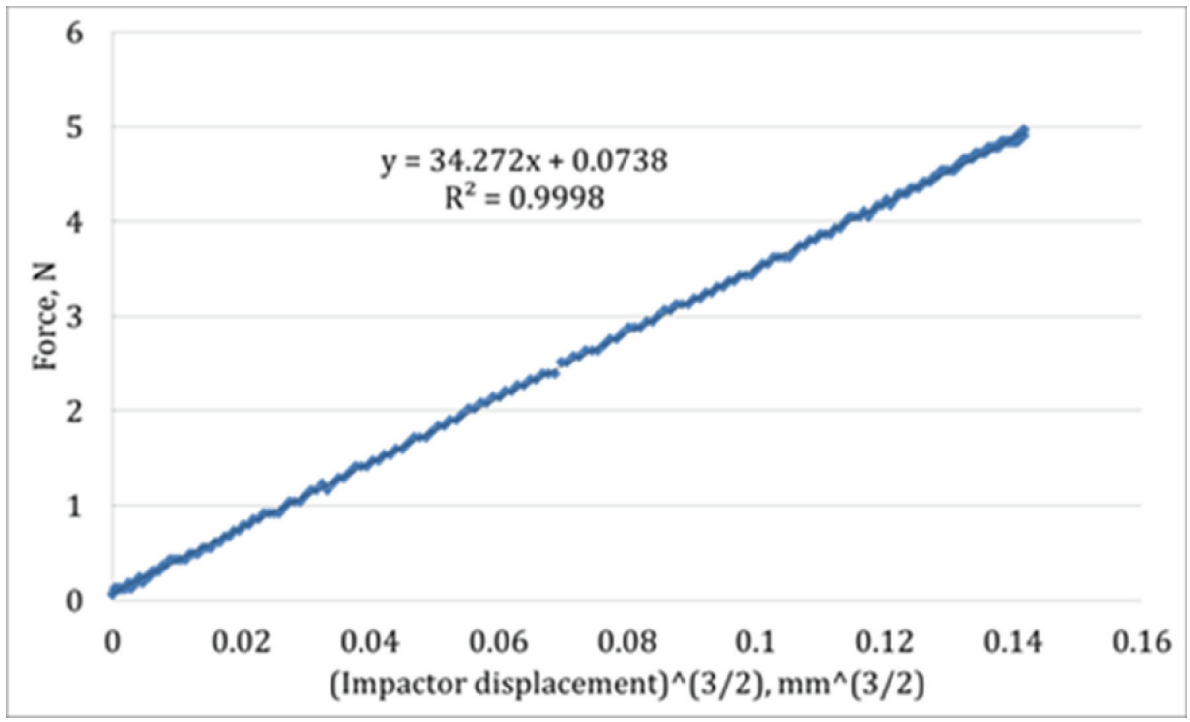

(b)

Figure 13. The Force vs Deformation curve (a) and the Hertz-interpretation (b), calculated according to the Eq. (7) and Eq. (8) (sample: nectarine) 


\subsection{Repeatability and reproducibility}

The repeatability tests are illustrated in Fig. 14 for a model material (a) and for a horticultural produce (b). The model material in this case was a soft silicone block (flat surface), the average DCE was $1.49 \pm 0.03 \mathrm{MPa}$ (the Coefficient of Variation is $1.2 \%$ ). The $\mathrm{CV} \%$ parameter characterizes the repeatability of the instrument and the method. It was typical for all model materials tested (rubber and elastic foam balls, etc.), resulting in CV\% around 1-3\% (it is in accordance with the literature). It shows a good/very good repeatability of the measurement. It is a little bit poorer than the extreme repeatability of the acoustic response method $(0.1-0.5 \%$, Felföldi and Fekete (2003)), but good enough for monitoring applications.

A difference in behavior during repeated tests can be recognized in case of samples of biological origin (illustrated by a tomato sample in Figure 14 (b)). The small scale (virtual) hardening after the first impact is very typical for all tested fruits and vegetables. The microscopic evaluation of possible bruise showed no destruction even after 10-20 repeated tests. It is emphasized by the physical parameters of the impact: the penetration depth was between $0.1-0.3 \mathrm{~mm}$ during the investigations, which is much below the bioyield deformation of the samples; the kinetic energy of the impactor (0.1-1 mJ) corresponds with falling of the sample from several tenth of millimeter. Conclusively, we supposed that no destruction was caused by the impact test with the given instrumentation and setup. According to our approach, realignment in the intracellular water content can happen due to the impact, resulting in some changes in mechanical properties, increasing of the DCE parameter. It was verified by repetition of the tests (on the very same location) after a relaxation period: the time-consuming water diffusion tends to reconstruct the original state.

The repeatability (of the instrument and the method) in this case can be characterized by the CV\% of the tests from the $2^{\text {nd }}$ impact. Results of some produces are summarized in Table 1.

Table 1. Mechanical characteristics of the tested cultivars (average of 20-20 samples)

\begin{tabular}{|c|c|c|c|c|c|}
\hline \multicolumn{1}{|c|}{ Tested sample } & Apple & Nectarine & Tomato & Bell pepper & Cucumber \\
\hline $\begin{array}{l}\text { Dynamic Coefficient of } \\
\text { Elasticity, MPa }\end{array}$ & 28.24 & 5.12 & 2.36 & 3.02 & 36.33 \\
\hline Coefficient of Variation, \% & 0.83 & 1.15 & 1.56 & 2.06 & 1.63 \\
\hline
\end{tabular}




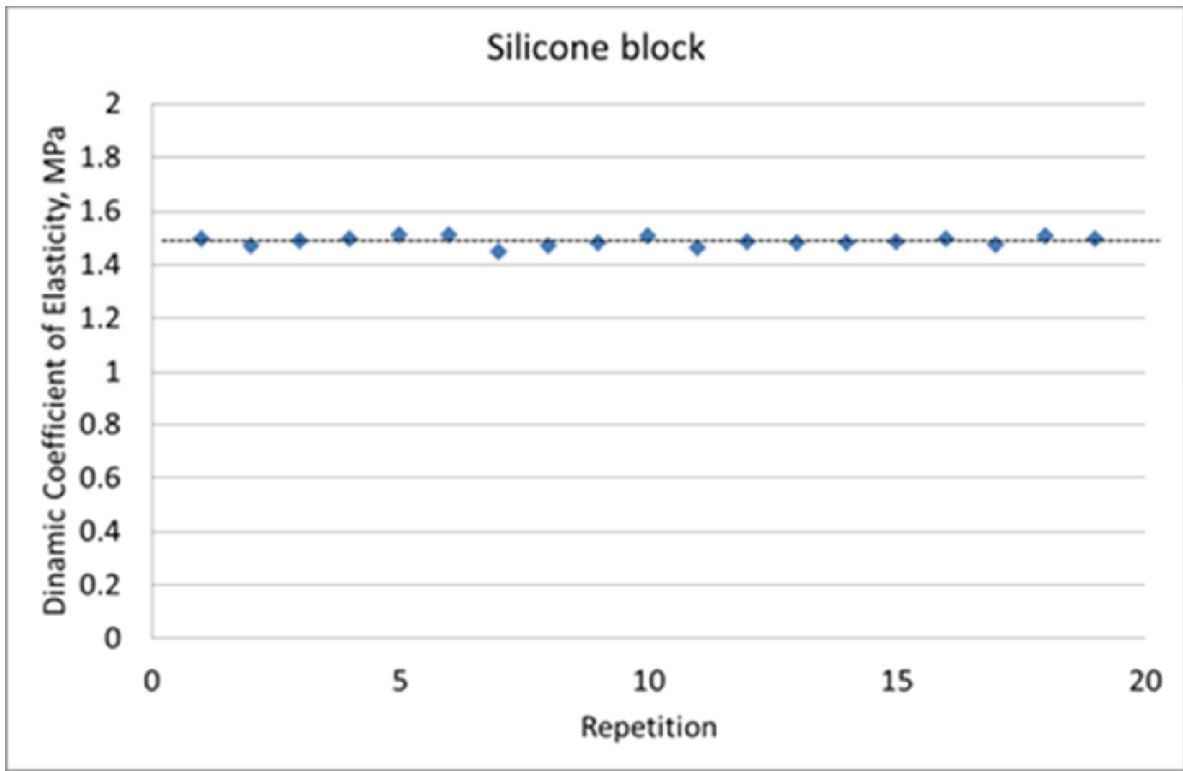

(a)

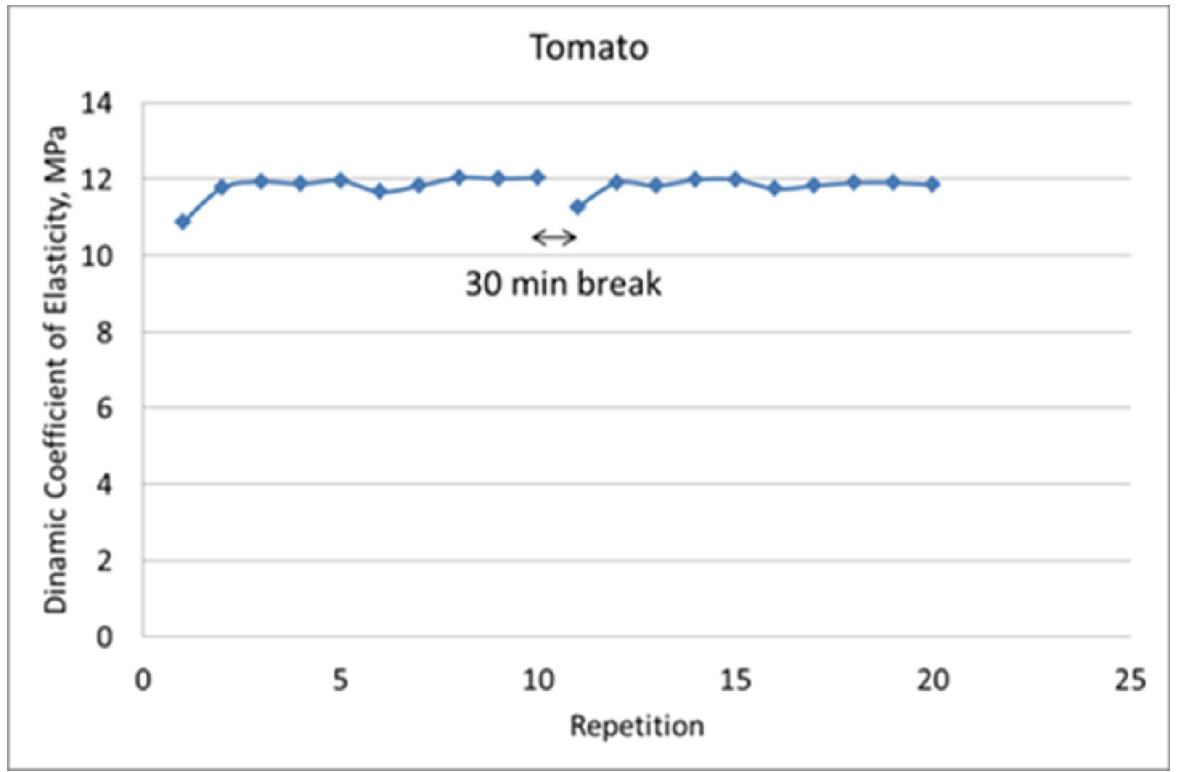

(b)

Figure 14. Repeatability tests on a Silicone block model material (a), and on a tomato sample, as a horticultural produce (b) 
The reproducibility tests (repeated impacts on a sample on different locations, e.g. around the equator) resulted - of course - in much higher CV\%-s (5 to $20 \%$, depending on the cultivar). It draws our attention to the necessity of the repeated test on a sample in order to get a representative characteristic of the mechanical stage despite the high biological variability of the samples.

\subsection{Application examples}

Due to the good repeatability and the non-destructive nature, the developed instrumentation seems to be suitable for monitoring of small scale changes. It was tested on cucumber samples (10 pcs), measured from the harvest (within 10 minutes) at room temperature in a 180-minute long period. The results are illustrated in Figure 15.

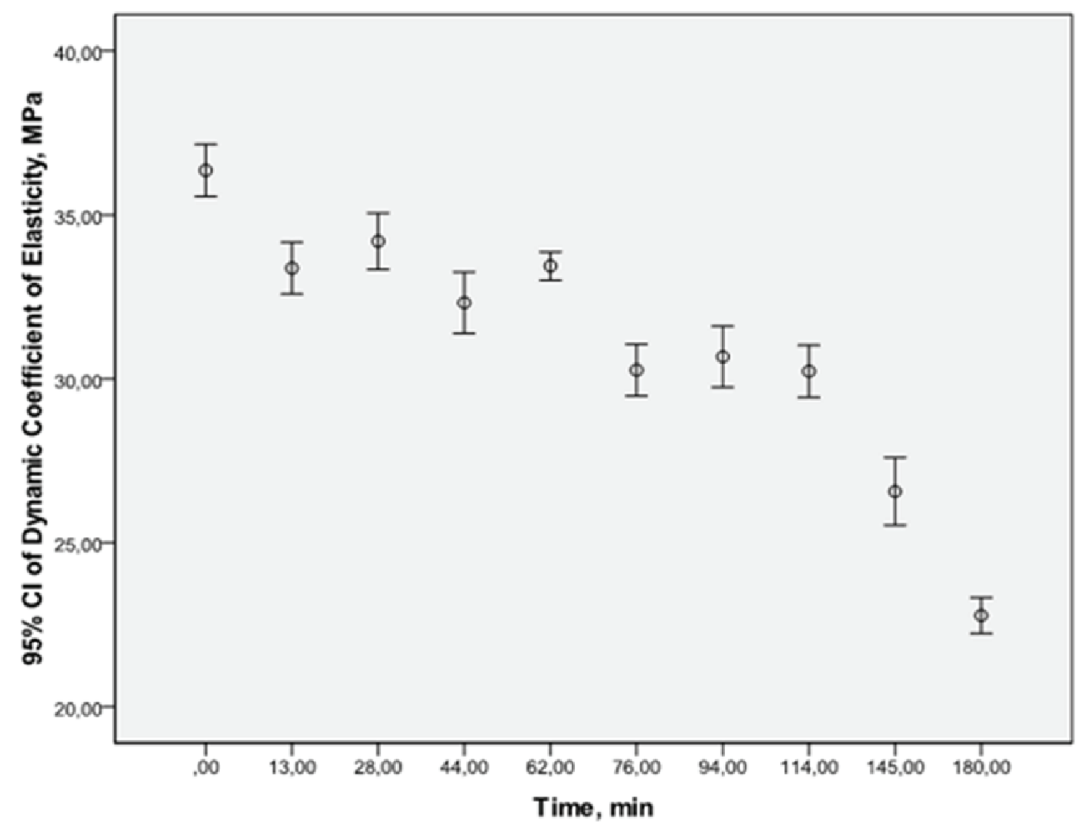

Figure 15. Firmness change of cucumber samples after harvesting (95\% Confidence Interval for Mean)

The very intensive softening of the cucumber after harvesting ( $40 \%$ in 3 hours $)$ is due to the intensive water loss at room conditions $\left(22{ }^{\circ} \mathrm{C}, 65 \%\right.$ $\mathrm{RH})$. Due to the sensitivity and repeatability of the method, significant 
change in mechanical properties can be detected even after 10-20 minutes. It means that it can be very useful in modeling of the processes, comparison of different cultivars, treatment or storage methods, etc.

Applying the models (resulted by the differential equation based numerical simulations), apple samples were tested to verify the viscoelastic behavior of real - horticultural - samples. Figure 16 demonstrates the $\mathrm{F}(\mathrm{t})$ and $x(t)$ curves of the selected sample are highlighting the $\mathrm{dT}_{\mathrm{v}=0}$ and $\mathrm{dT}_{\mathrm{Fmax}}$ parameters. The remarkable difference between their values shows relatively high viscosity of the sample. According to the regression models in Fig. 9 and Fig. 10, E = 8.2 Mpa and $c=4.10^{5} \mathrm{Pas} / \mathrm{m}$ values were estimated for the Young's modulus and the viscosity constant of the given sample, respectively (slightly above the validity range of the viscosity model).

The shape of the curve on the Force vs. Deformation diagram (Fig. 17a) validates this level of the viscoelasticity of the sample, comparing it with Figure 8. Applying the Hertz model, linear connection was expected between the force and the "deformation on the (3/2) power" according to Eq. (1). The high determination coefficient in Fig. $17 b$ validates our approach. The slope of the initial linear interval can be used to determine the Dynamic Coefficient of Elasticity according to Eq. (10) (DCE = 9.41 $\mathrm{MPa}$ for the demonstrated apple sample).

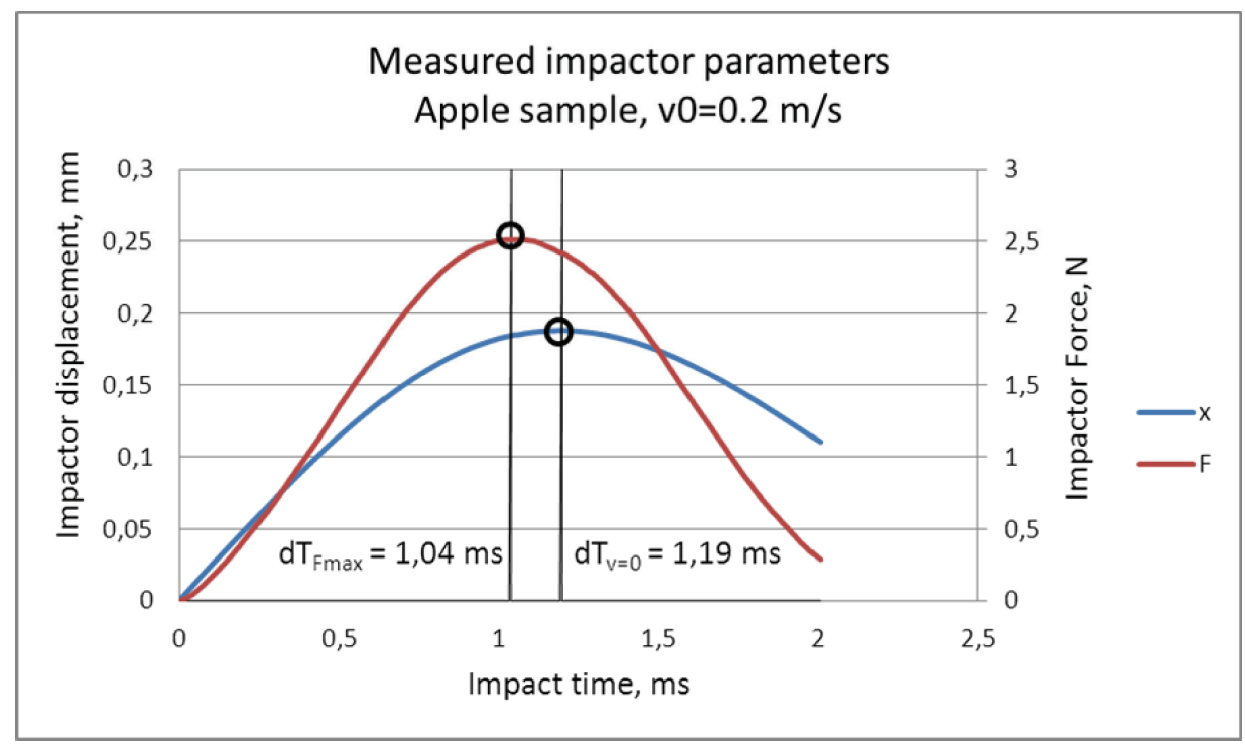

Figure 16. Measured (F) and calculated (x) impact characteristic (sample: apple), with the time-to-the-peak parameters 


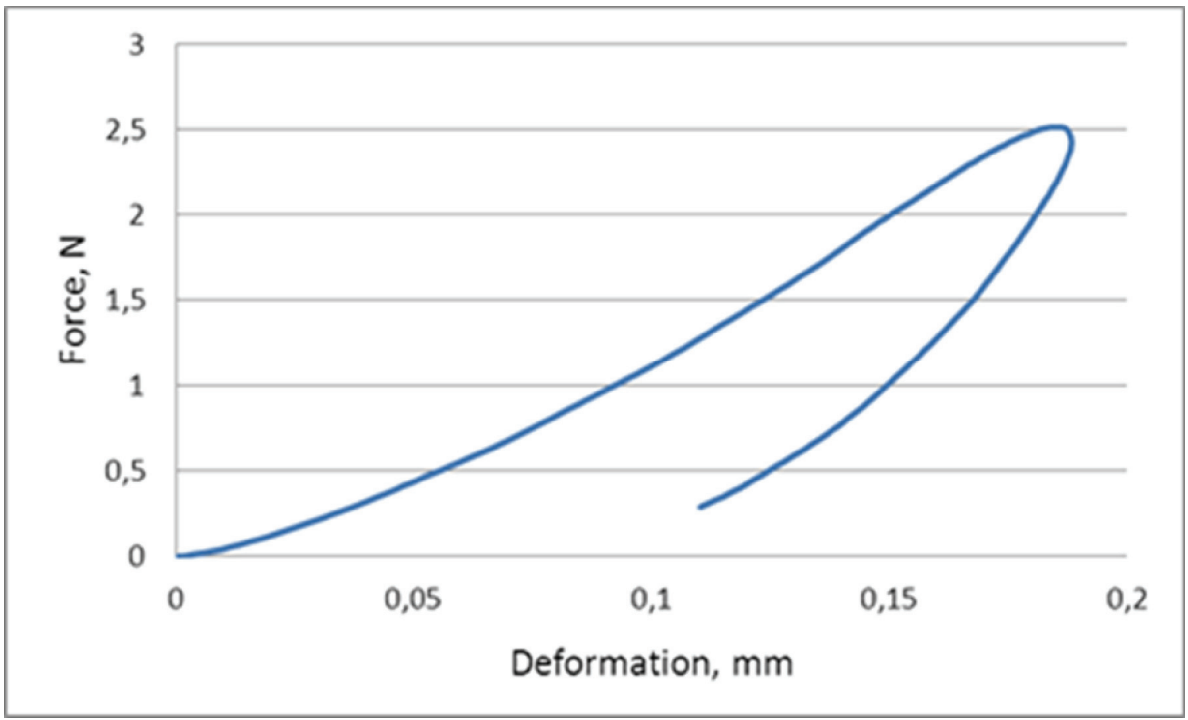

(a)

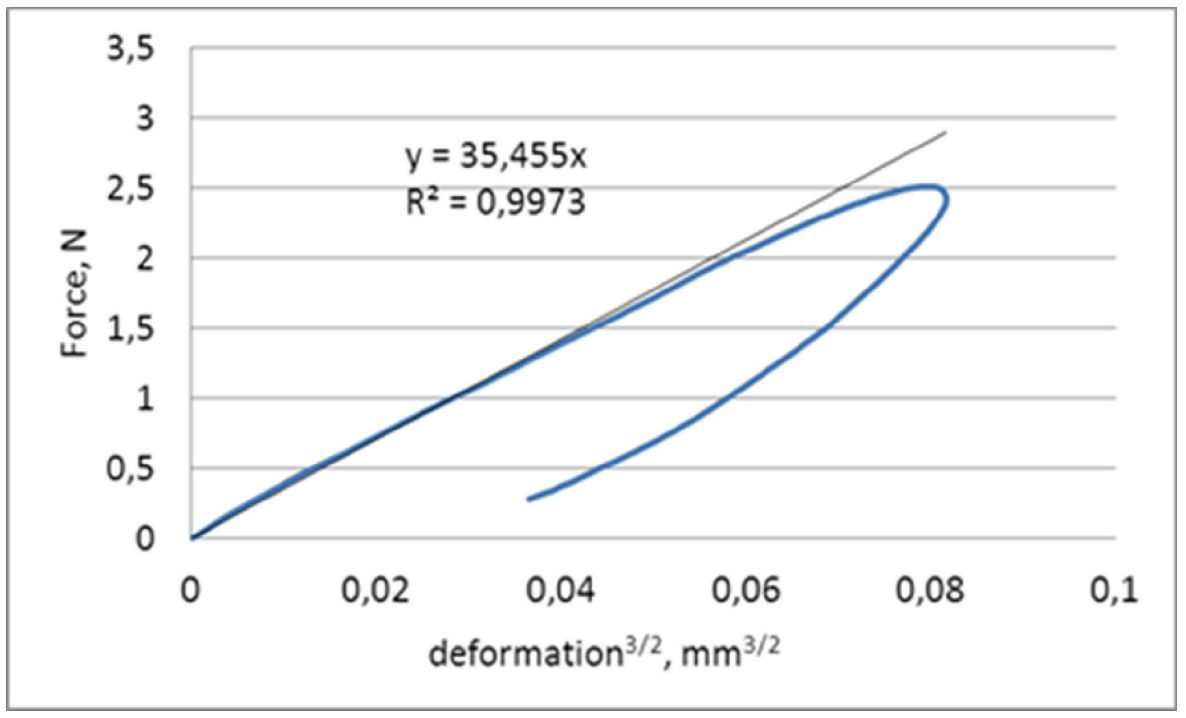

(b)

Figure 17. The force-deformation curve and the same result in the Hertzinterpretation 


\section{Conclusions}

The developed instrumentation was found to be able to measure the surface mechanical properties of wide range of fruits and vegetables nondestructively, with very good repeatability. Due to its construction and nondestructive nature, it is suitable for in-vivo application as well, offering an especially useful tool for organic horticulture in maturation monitoring, treatment evaluation, defect detection as well. It is sensitive and accurate enough to detect and measure even the small scale changes during the maturation and postharvest handling of the samples.

New mechanical characteristic (Dynamic Coefficient of Elasticity) was introduced, connected directly to the Young's modulus (E). The advantage of the physical interpretation of the process and determining mechanical parameters in physical dimensions instead of empirical indices is obvious (calibration transfer, comparability of different instrument, different setups, etc.).

Further investigations are needed for comparison of the results with other dynamic and traditional methods. Hardware development is necessary to measure the impactor speed as well for the correct interpretation of the results in case of characteristically viscoelastic materials as well.

\section{References}

Cheng-Chang Lien, Ching-Hua Ting (2014) Assessing guava maturity by statistical analyses of dropped fruit impact responses. Postharvest Biology and Technology, 95, 20-27.

De Belie, N., S. Schotte, J. Lammertyn, B. Nicolai, J. De Baerdemaeker (2000) Firmness changes of pear fruit on the tree with the acoustic impulse response technique. AgEng, Warwick 2000, Paper Number 00-PH-001.

Felföldi J, T. Ignát. (1999) Dynamic method for quick and non-destructive measurement of the surface firmness of fruits and vegetables. Hungarian Agricultural Engineering $12,29-30$.

Felföldi, J. and A. Fekete (2003) Detection of small scale mechanical changes by acoustic measuring system - ASAE Annual Meeting Paper, Las Vegas, No. 036097, pp. 1-8.

Felföldi, J. and V. Muha (2007) In-vivo acoustic measurement of mechanical properties of fruits -International Conference on Agricultural, Food and Biological Engineering \& Post Harvest/Production Technology, Khon Kaen - Paper No. 61, pp. 1-7.

Garcia-Ramos, F.J., J Ortiz-Cañavate, M. Ruiz-Altisent, J. Díez, L. Flores, I. Homer, J.M. Chávez (2003) Development and implementation of an on-line impact sensor for firmness sensing of fruits. Journal of Food Engineering, 58(1), 53-57. 
Mizrach, A. (2000) Determination of avocado and mango fruit properties by ultrasonic technique. Ultrasonics, 38(1-8), 717-722.

Mohsenin, N. N. (1986). Physical properties of plant and animal materials. Gordon and Breach Science Publishers, London.

Muha, V., J. Felföldi (2005) Methodological examinations of dynamic texture measurement. Journal of Food Physics XVII-XVIII, 3-12.

Padungsak Wanitchang, Anupun Terdwongworakul, Jaitip Wanitchang, Natrapee Nakawajana (2011) Non-destructive maturity classification of mango based on physical, mechanical and optical properties. Journal of Food Engineering, 105(3), 477-484.

Rodriguez-Sinovas, L., M. Ruiz-Altisent and J.L. de la Plaza Perez (1991) Bruise development and fruit response of pear (cv. Blanquilla) under impact conditions. Journal of Food Engineering, 14, 289-301.

Zsom-Muha, V. and J. Felfoldi (2007) Vibration Behavior of Long Shape Vegetables Progress in Agricultural Engineering Sciences, 3, 21-46. 